\title{
Mammalian target of rapamycin signaling is involved in the vasculogenic mimicry of glioma via hypoxia-inducible factor-1 $\alpha$
}

\author{
MIN HUANG ${ }^{1}$, YIQUAN KE ${ }^{1}$, XINLIN SUN ${ }^{1}$, LI YU $^{2}$, ZHILIN YANG $^{1}$, YONGHONG ZHANG $^{1}$, \\ MOUXUAN DU ${ }^{1}$, JIHUI WANG ${ }^{1}$, XIAO LIU $^{1}$ and SHUYUN HUANG ${ }^{1}$ \\ ${ }^{1}$ The National Key Clinic Specialty, The Neurosurgery Institute of Guangdong Province, \\ Guangdong Provincial Key Laboratory on Brain Function Repair and Regeneration, Department of Neurosurgery, \\ Zhujiang Hospital, Southern Medical University; ${ }^{2}$ Department of Pathology, Zhujiang Hospital, \\ Southern Medical University, Guangzhou, Guangdong 510280, P.R. China
}

Received July 11, 2014; Accepted August 14, 2014

DOI: 10.3892/or.2014.3454

\begin{abstract}
The mammalian target of rapamycin (mTOR) is a crucial regulator in malignant gliomas. Vasculogenic mimicry (VM) describes functional channels established by highly malignant tumor cells that is different from endothelium-lined blood vessels. Our previous studies confirmed the existence and clinical significance of VM in medulloblastoma and glioblastoma. In the present study, by immunohistochemical and CD34/PAS histochemical double-staining, 34 cases (26.8\%) with VM structures were identified among a total of 127 glioma cases, and these VM structures were associated with mTOR expression in the glioma specimens. In vitro, U87 malignant glioblastoma cells formed tube structures similar to HUVECs on Matrigel in 3D culture, and mTOR-specific inhibitor rapamycin inhibited VM formation in the U87 malignant glioblastoma cells under both normoxia and hypoxia. In addition, rapamycin and mTOR siRNA inhibited molecules in the signaling cascade of VM formation, particularly HIF-1 $\alpha$. Taken together, our results demonstrated that mTOR signaling is involved in VM formation, and may be a potential therapeutic target for gliomas.
\end{abstract}

\section{Introduction}

Glioma is one of the most common malignant primary brain tumors. According to the 2007 World Health Organization classification of central nervous system tumors, gliomas are divided pathologically into four grades, which are relevant for prognosis. Surgery, with or without radiotherapy and chemotherapy, can relieve many symptoms in glioma patients,

Correspondence to: Professor Yiquan Ke, Department of Neurosurgery, Zhujiang Hospital, Southern Medical University, 253 Gongye Road, Guangzhou, Guangdong 510280, P.R. China E-mail: zjkeyiquan@gmail.com

Key words: glioma, hypoxia-inducible factor-1 $\alpha$, mammalian target of rapamycin, vascular-targeted therapy, vasculogenic mimicry but clinically, tumor recurrence often occurs (1). For many patients, complete elimination of gliomas remains a challenge.

In recent years, vascular-targeted therapy has gradually been accepted. The antiangiogenic drug bevacizumab (Avastin) has become one of the most popular vascular-targeted therapeutic drugs for several types of tumors, including gliomas (2). However, this traditional antiangiogenic drug also accelerates metastasis, together with marked hypoxia and an alternative blood supply - vasculogenic mimicry (VM) (3). VM consists of laminin-rich networks that can be stained with periodic acid-Schiff (PAS) in vivo and forms extracellular matrix (ECM)-rich tubular networks on Matrigel that mimic conventional angiogenesis in vitro. VM is established by highly aggressive tumor cells instead of poorly aggressive ones or endothelial cells (4). Our previous studies confirmed the existence and clinical significance of VM in medulloblastoma and glioblastoma, and VM might be an independent adverse prognostic factor for overall survival $(5,6)$. As a novel form of blood supply, suppression of VM could be an alternative therapeutic target for gliomas.

The mammalian target of rapamycin (mTOR) signaling pathway is activated in the majority of human tumors. It plays an important role in regulating angiogenesis both in normal tissues and in tumors (7). Previous studies have demonstrated that there is molecular crosstalk between the mTOR and VM signaling pathways, particularly HIF-1 $\alpha$ (8).

A recent study indicated that rapamycin acts as an HIF-1 $\alpha$ inhibitor and prevents VM formation of human epithelial ovarian cancer in vivo (9). Researchers have reached a consensus that HIF-1 $\alpha$ is a major cause of VM (reviewed in ref. 10). Therefore, HIF-1 $\alpha$ might be one of the mTOR downstream molecules involved in VM. However, the integrated mechanism of mTOR signaling in VM formation has not yet been investigated. In this study, we aimed to achieve a better understanding of the role of mTOR signaling in VM formation and to explore a novel method of treatment targeting glioma.

\section{Materials and methods}

Patients. One hundred and twenty-seven specimens of paraffin-embedded glioma tissues were obtained from the 
Department of Pathology of Zhujiang Hospital at Southern Medical University between 2009 and 2012. Tumor sections were reviewed by two neuropathologists to verify the diagnosis of glioma in accordance with the 2007 World Health Organization classification of central nervous system tumors. Informed consent was obtained for the use of the specimens, and the study was approved by the Research Ethics Committee of Southern Medical University.

Cells and reagents. The human $\mathrm{U} 87$ malignant glioblastoma (U87-MG) cell line was chosen for the in vitro functional test, and human umbilical vein endothelial cells (HUVECs) were used as control cells. Both cell lines were cultured in Dulbecco's modified Eagle's medium (DMEM; Hyclone, Logan, UT, USA) supplemented with $10 \%$ fetal bovine serum (FBS; Gibco, Minneapolis, MN, USA). Cells were grown at $37^{\circ} \mathrm{C}$ in a humidified atmosphere of $5 \% \mathrm{CO}_{2}$ and $95 \%$ air. Serum-free culture medium was used for the cell function assays.

Rapamycin (Sigma-Aldrich, St. Louis MO, USA) was stored at a concentration of $10 \mathrm{mM}$ in $100 \%$ DMSO at $-20^{\circ} \mathrm{C}$ and was diluted in serum-free medium immediately prior to use. Antibodies against mTOR, HIF-1 $\alpha$, MMP-14 and MMP-2 were purchased from Abcam (Cambridge, UK).

Hypoxia treatment. Hypoxic conditions were simulated by flushing $5 \% \mathrm{CO}_{2}$ and $95 \% \mathrm{~N}_{2}$ through a modified chamber (Mitsubishi, Japan), as described previously (11), until the $\mathrm{O}_{2}$ concentration was reduced to $1 \%$, as measured with a Mini oxygen meter. The culture system was sealed and incubated at $37^{\circ} \mathrm{C}$.

Immunohistochemical and CD34/PAS histochemical double-staining. Immunohistochemical and CD34/PAS histochemical double-staining were performed as we previously described (6). For immunohistochemical staining of each slide, a total of five random images (magnification, $\mathrm{x} 400$ ) were selected and examined under a microscope (Leica, Germany). The number of stained cells and the total number of cells were counted, and the ratio between the stained and total cells was calculated. The following scoring was used for the stained cell ratio: $<10 \%$ was negative or weakly positive $(-/+) ; 10-50 \%$ was strongly positive $(++)$; and $>50 \%$ was very strongly positive $(+++)$.

For CD34/PAS histochemical double-staining, after immunohistochemical staining for CD34 (Zhongshan Goldenbridge Biotechnology, Beijing, China), the sections were washed with distilled water and slides were stained following the PAS staining procedures before counterstaining with Mayer's hematoxylin.

Three-dimensional (3D) culture. The in vitro study of vasculogenic mimicry formation was assessed on Matrigel by a 3D culture. Matrigel (Growth Factor Reduced; BD Biosciences) was thawed at $4^{\circ} \mathrm{C}$, and $250 \mu 1$ was quickly added to each well of a 24-well plate and allowed to solidify for $1 \mathrm{~h}$ at room temperature. HUVECs or U87-MG cells were harvested by trypsin, resuspended in medium with serum in the presence or absence of rapamycin at the indicated concentrations, and seeded onto the Matrigel layer at $1-5 \times 10^{4}$ cells/well. The
Table I. Relationship between VM and clinicopathological data of the patients with glioma.

\begin{tabular}{|c|c|c|c|c|c|}
\hline \multirow[b]{2}{*}{ Variables } & \multirow[b]{2}{*}{ Cases } & \multicolumn{2}{|c|}{ VM } & \multirow[b]{2}{*}{$\chi^{2 \mathrm{a}}$} & \multirow[b]{2}{*}{ P-value } \\
\hline & & Positive & Negative & & \\
\hline Gender & & & & 0.486 & 0.486 \\
\hline Male & 72 & 21 & 51 & & \\
\hline Female & 55 & 13 & 42 & & \\
\hline Age (years) & & & & 0.011 & 0.917 \\
\hline$<60$ & 85 & 23 & 62 & & \\
\hline$\geq 60$ & 42 & 11 & 31 & & \\
\hline KPS & & & & 0.026 & 0.872 \\
\hline$<60$ & 36 & 10 & 26 & & \\
\hline$\geq 60$ & 91 & 24 & 67 & & \\
\hline Tumor size $(\mathrm{cm})$ & & & & 0.132 & 0.717 \\
\hline$<6$ & 78 & 20 & 58 & & \\
\hline$\geq 6$ & 49 & 14 & 35 & & \\
\hline Pathological grade & & & & 9.051 & $0.029^{\mathrm{b}}$ \\
\hline I & 7 & 0 & 7 & & \\
\hline II & 45 & 7 & 38 & & \\
\hline III & 42 & 14 & 28 & & \\
\hline IV & 33 & 13 & 20 & & \\
\hline mTOR expression & & & & 7.748 & $0.021^{\mathrm{b}}$ \\
\hline$-/+$ & 10 & 1 & 9 & & \\
\hline++ & 83 & 18 & 65 & & \\
\hline+++ & 34 & 15 & 19 & & \\
\hline
\end{tabular}

${ }^{\text {a Pearson }} \chi^{2}$ test (asymptotic significance, two-sided). ${ }^{\text {b }} \mathrm{P}<0.05$ indicates a significant difference. KPS, Karnofsky performance score.

HUVECs were used as a control. After a 6-h incubation, the tubular network structures were visualized, and images were captured under a phase contrast microscope. The relative lengths of the tubes were quantified by image analysis software (Image-Pro Plus).

Western blotting. The cells were lysed with RIPA buffer (Beyotime, Nangtong, China) with the addition of $1 \%$ fresh protease inhibitor cocktail 1 and/or phosphatase inhibitor cocktail 2 (Sigma) and $1 \mu 1100 \mathrm{mM}$ phenylmethylsulfonyl fluoride (Beyotime). After removal by scraping, the cells were placed in ice for $30 \mathrm{~min}$ and centrifuged at $12,000 \mathrm{rpm}$ for $10 \mathrm{~min}$. The protein concentration of the samples was determined using an Enhanced BCA protein assay kit (Beyotime). The protein concentrations were quantified and $30 \mu \mathrm{g}$ protein per sample was separated on $8 \%$ SDS-PAGE. The separated proteins were transferred onto polyvinylidene difluoride membranes (Millipore). After blocking with 5\% non-fat milk for $1 \mathrm{~h}$ at room temperature, the membranes were probed with primary antibodies for $2 \mathrm{~h}$ at room temperature, followed by appropriate horseradish peroxidase-conjugated secondary antibodies (all from Abcam) for $1 \mathrm{~h}$ at room temperature. The blots were detected using Pierce ECL Plus Western Blotting Substrate (Thermo Fisher) and developed using X-ray film. 
A

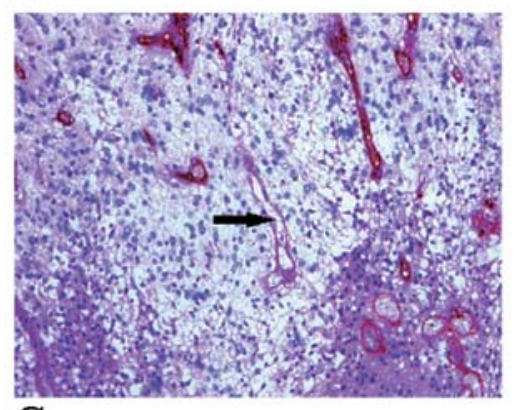

C

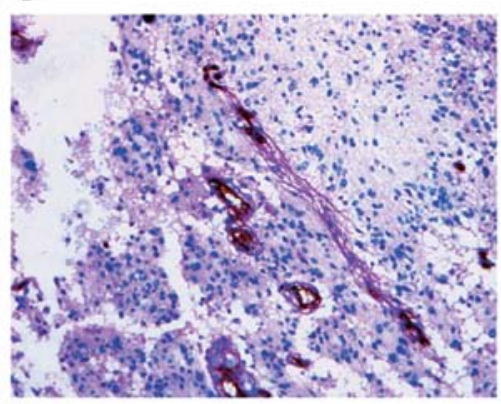

E

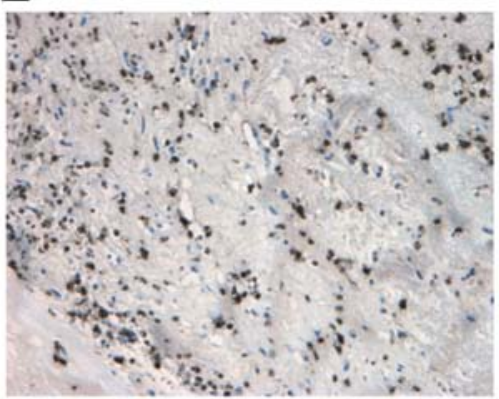

B

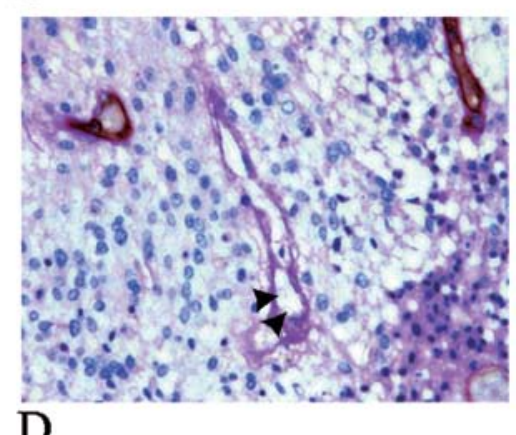

D

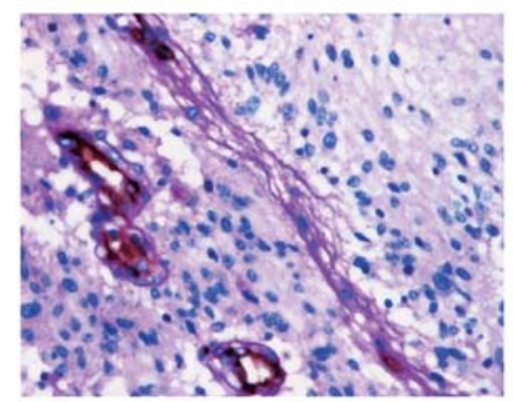

F

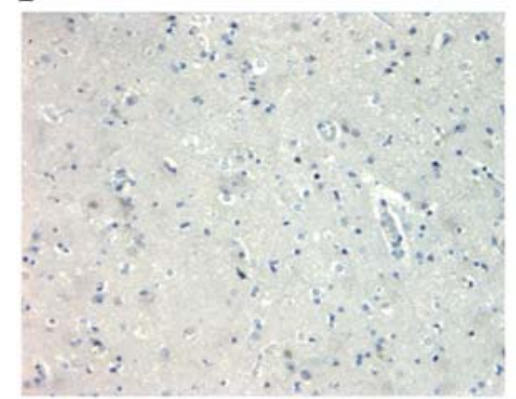

Figure 1. Immunohistochemistry of VM structures and mTOR expression in the glioma tissues. (A) Representative VM structure (arrow) and endothelial cell-lined blood vessels (brown staining) in glioma tissues. The VM structure was stained purple with PAS, and endothelial cell-lined blood vessels were stained brown. (B) Higher magnification of the VM structure containing red blood cells (arrow heads). (C and D) Some VM structures were connected with CD34-positive endothelial cell-lined blood vessels. mTOR expression in the (E) VM group of glioma tissues was significantly higher than that in the (F) non-VM group. Original magnification: x200 in A, C, E and F; x400 in B and D. Tissue stains: CD34/PAS histochemical double-staining in A-D; immunohistochemical staining in $\mathrm{E}$ and $\mathrm{F}$.

siRNA transfection. The negative control and mTOR siRNAs were purchased from GenePharma Biological Technology (Shanghai, China). The target sequence of mTOR siRNA was 5'-GGCCUAUGGUCGAGAUUUATT-3' (12). For siRNA transfection, cells at a concentration of $2.5-5 \times 10^{4}$ cells $/ \mathrm{ml}$ were incubated for $24 \mathrm{~h}$ in 6 -well plates. The cells were then transfected with 200 pmol negative control (NC) and mTOR siRNA for 24-48 hin the presence of Lipofectamine(Invitrogen) and subjected to western blotting. We also used a positive control (GAPDH siRNA) and a fluorescein-labeled negative control to ensure the reliability of the method and transfection efficiency.

Cell migration assay. Cell migration was evaluated using an in vitro wound healing assay. Cells were seeded on a 6-well plate and incubated for $6 \mathrm{~h}$ to allow the formation of a cell monolayer. Cells were scratched with the tip of a $200-\mu 1$ pipette and then incubated at $37^{\circ} \mathrm{C}$ under normoxic or hypoxic conditions for $24 \mathrm{~h}$. Cell motility was assessed by measuring the speed of wound closure at specific intervals. Each experiment was conducted in triplicate.

Statistical analysis. All experiments were repeated at least three times. The data are expressed as mean \pm standard deviation (SD) or standard error of the mean (SEM). Statistical analysis was performed using the Student's t-test (two-sided). The criterion for statistical significance was $\mathrm{P}<0.05$ or $\mathrm{P}<0.01$.

\section{Results}

Relationship between VM and clinicopathological data of the glioma cases. Thirty-four cases (26.8\%) with VM structures were identified among a total of 127 glioma cases (Table I). These structures were positive for PAS but negative for CD34 (Fig. 1A), indicating that they did not consist of endothelial cells. Red blood cells were observed at higher magnification in the VM structures (Fig. 1B) suggesting that they had a blood supply function. Some VM structures were even 
A

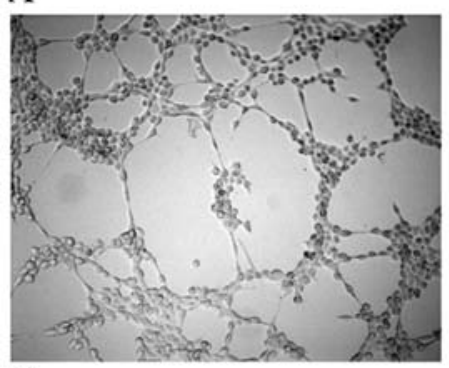

$\mathrm{C}$

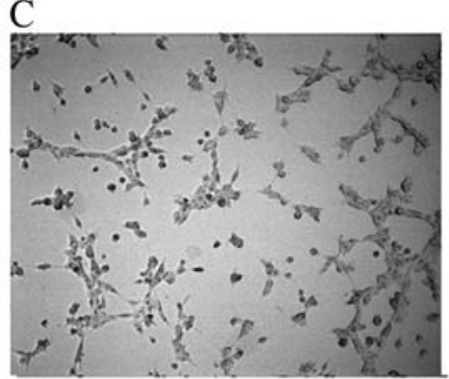

B

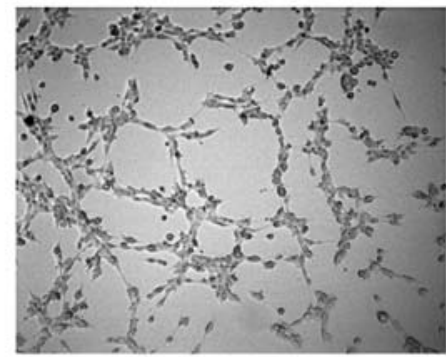

$\mathrm{D}$

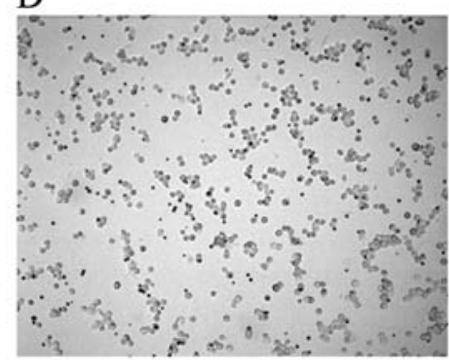

$\mathrm{E}$

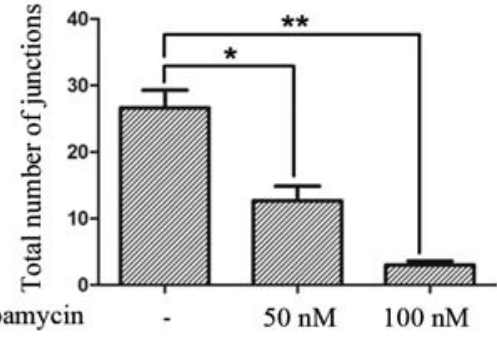

Figure 2. Inhibition of tube formation in U87-MG cells by rapamycin under normoxic conditions. (A) Representative image of tube structure formation in HUVECs on Matrigel (magnification, x100). (B-D) U87-MG cells formed defective tube structures on Matrigel. Cells treated with (C) $50 \mu \mathrm{M}$ or (D) $100 \mu \mathrm{M}$ rapamycin showed different degrees of tube structure damage. (E) Total number of junctions per field in x100 view was compared between groups. Data represent the mean \pm SEM from three independent experiments. ${ }^{*} \mathrm{P}<0.05$ and ${ }^{* *} \mathrm{P}<0.01$ compared with the group without addition of rapamycin.

interlinked with $\mathrm{CD}^{+} 4^{+}$endothelial cell-lined blood vessels (Fig. 1C and D).

Among all of the clinicopathological variants compared, the pathological grade of gliomas and the mTOR expression in the tissue sections differed significantly between the VM-positive and VM-negative group $(\mathrm{P}<0.05)$. However, there was no relationship with other clinicopathological variants such as gender, age, Karnofsky performance score (KPS) and tumor size.

VM structures were found more frequently in highly aggressive gliomas (33.3\% of grade III and $39.4 \%$ of grade IV cases) compared to the poorly aggressive ones $(15.6 \%$ of grade II and $0.0 \%$ of grade I cases $)\left(\chi^{2}=9.051, \mathrm{P}=0.029\right)$, which is consistent with other tumors from different studies $(4,13)$.

mTOR protein expression in the human glioma tissues was investigated by immunohistochemistry (Fig. 1E and F). VM structures were significantly more frequent in the tissues with a higher rate of mTOR expression than the frequency of VM structures in tissues with a lower rate of mTOR expression $(10.0 \%$ of $-/+, 21.7 \%$ of ++ and $44.1 \%$ of +++ tissues $)\left(\chi^{2}=7.748\right.$; $\mathrm{P}=0.021)$. These results strongly imply that $\mathrm{VM}$ is not only correlated with tumor grade but also with mTOR signaling activity in gliomas.

Rapamycin inhibits tube structures in the U87-MG cell line under normoxia. In the in vitro test, under normoxia, U87-MG cells formed tube structures similar to HUVECs on Matrigel (Fig. 2A and B). The tube structures were significantly inhibited with increasing concentrations of rapamycin (Fig. 2C and D), and there was a significant difference between the various degrees of inhibition (Fig. 2E).

Rapamycin inhibits stronger tube structures in the U87-MG cell line under hypoxia. As showed in the previous experiment, the ability of U87-MG cells to form tube structures on Matrigel appeared defective under a normoxic condition (Fig. 3A). The tumor cells grown under hypoxic conditions showed stronger tube structures on Matrigel (Fig. 3B). However, when treated with rapamycin, these tube structures disappeared (Fig. 3C and D).

Rapamycin inhibits mTOR and HIF-1 $\alpha$ expression under normoxic or hypoxic conditions. Many studies have confirmed that intratumoral hypoxia is closely related with the formation of VM, and HIF-1 $\alpha$ is often activated $(14,15)$. We first examined the effect of rapamycin on the activation of HIF-1 $\alpha$ under normoxic conditions. Western blotting revealed that rapamycin inhibited HIF-1 $\alpha$ expression, even when it was expressed very low under normoxia (Fig. 4A). Quantitative analyses of the western blotting results revealed that treatment with increasing concentrations of rapamycin induced a dose-dependent downregulation of HIF-1 $\alpha$ protein in addition to inhibiting mTOR expression (Fig. 4B). Additionally, rapamycin induced a dose-dependent downregulation of HIF-1 $\alpha$ expression under hypoxia. Inhibition of HIF-1 $\alpha$ accumulation by rapamycin (Fig. 4C) was similar to that in previous studies of exposure to hypoxia $(8,16)$.

mTOR siRNA significantly decreases downstream signaling of VM and suppresses glioma cell migration. To verify the regulation of downstream molecules of mTOR by rapamycin, U87-MG cells were transfected with mTOR siRNA. The efficiency and effectiveness of transfection were identified by western blot analysis. We designed and synthesized four mTOR siRNAs, and chose one that maximally knocked down mTOR expression, to screen the downstream signaling of VM. When mTOR expression was significantly decreased by siRNA, as expected, HIF-1 $\alpha$ expression was also significantly 
A

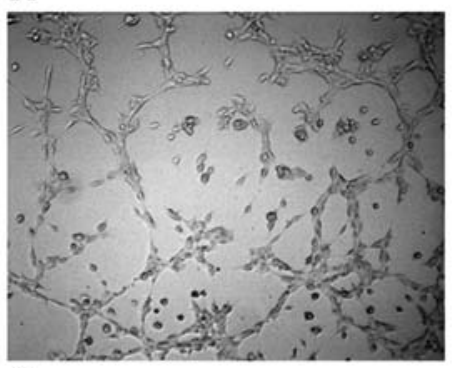

C

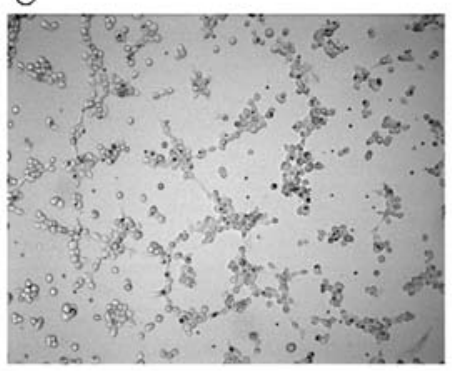

B

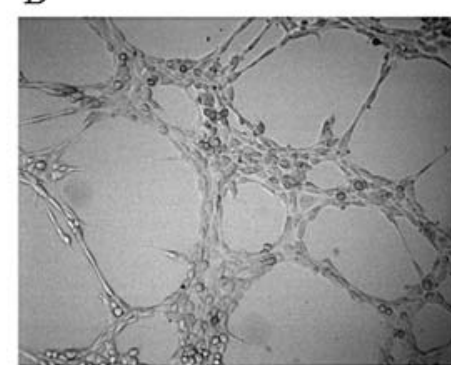

D

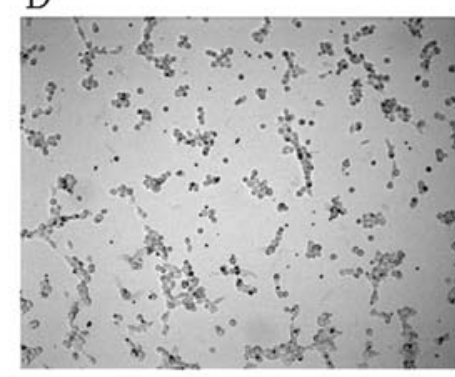

E

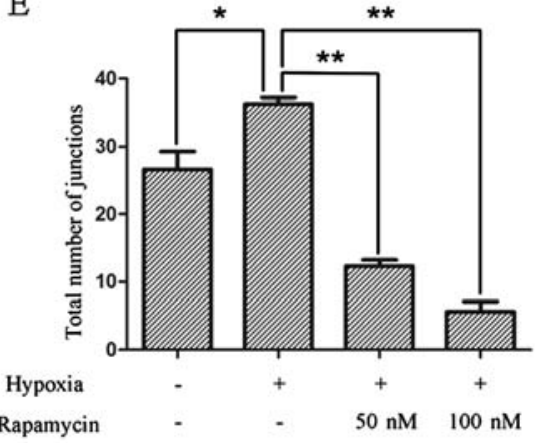

Figure 3. Inhibition of tube formation in U87-MG cells by rapamycin under hypoxic conditions. (A) U87-MG cells showed defective tube formation on Matrigel under normoxic conditions. (B) Under hypoxia, U87-MG cells grown on Matrigel formed stronger tube structures. In cells treated with (C) $50 \mu \mathrm{M}$ or (D) $100 \mu \mathrm{M}$ rapamycin, tube structures disappeared, even under hypoxia. (E) Total number of junctions per field in x100 view was compared between groups. Data represent the mean \pm SEM from three independent experiments. ${ }^{*} \mathrm{P}<0.05$ and ${ }^{* *} \mathrm{P}<0.01$ compared with the group without addition of rapamycin under hypoxia.

A

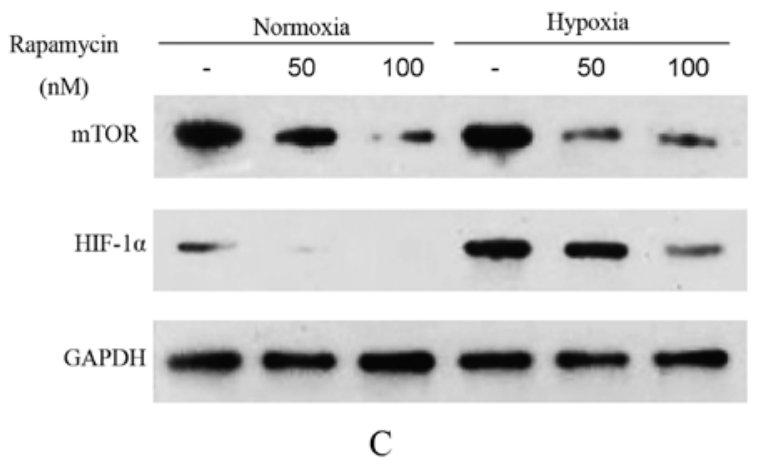

$\mathrm{B}$

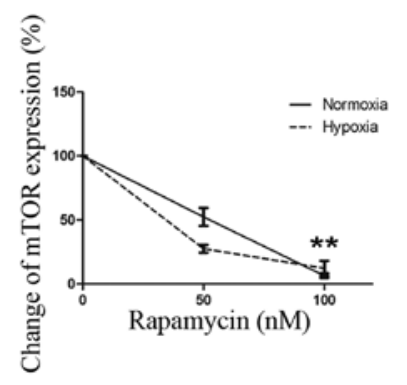

Figure 4. Effect of rapamycin on the expression of mTOR and HIF-1 $\alpha$. (A) Western blotting results revealed that rapamycin inhibits mTOR and HIF-1 $\alpha$ expression in the U87-MG cell line under normoxic or hypoxic conditions. (B and C) Quantitative analyses of the western blotting results indicate that treatment with increasing concentrations of rapamycin induced a dose-dependent downregulation of HIF-1 $\alpha$ protein in addition to inhibiting mTOR expression. ${ }^{*} \mathrm{P}<0.05,{ }^{* *} \mathrm{P}<0.01$ compared with the group without addition of rapamycin.

downregulated, under normoxic or hypoxic conditions (Fig. 5A). Quantitative analyses of the western blotting results

also showed the inhibitory effects of mTOR siRNA on the expression of related molecules (Fig. 5B).

Consequently, to establish whether the mTOR signaling pathway influenced the final stage of VM signaling, we investigated MMP-14 and MMP-2 expression by western blotting. As shown in Fig. 5A and B, expression of both MMPs was lower in the U87-MG cells transfected with mTOR siRNA than levels in the control cells, even under hypoxia.

MMP-2 is associated with cell migration (17). We further detected the migration of U87-MG cells when transfected with siRNA. Fig. 5C shows that cell migration increased after hypoxia for $24 \mathrm{~h}$. However, this increase did not recur after siRNA interference.

\section{Discussion}

The initial morphologic and molecular characterization of VM was accomplished in human melanoma. In addition to identification in invasive melanoma, VM has also been observed in other malignant solid tumors, including prostatic tumors $(18,19)$, Ewing sarcoma $(20)$, hepatocellular carcinoma $(21,22)$, colorectal carcinoma (23) and glioma (24). Our previous study demonstrated that VM exists in glioblastomas and is a significant prognostic factor for patient survival (5). In the present study, we confirmed that VM was present in different grades of glioma, and the amount of VM in the tumors increased with the grade of glioma. These results are consistent with a previous study that showed that VM formation is related to the invasive ability of tumors; more VM structures are observed in highly aggressive tumors than in less aggressive ones (4).

In addition to the malignancy of tumor cells and tumor blood supply, the surrounding microenvironment (such as hypoxia) is also closely related with VM formation $(25,26)$. 

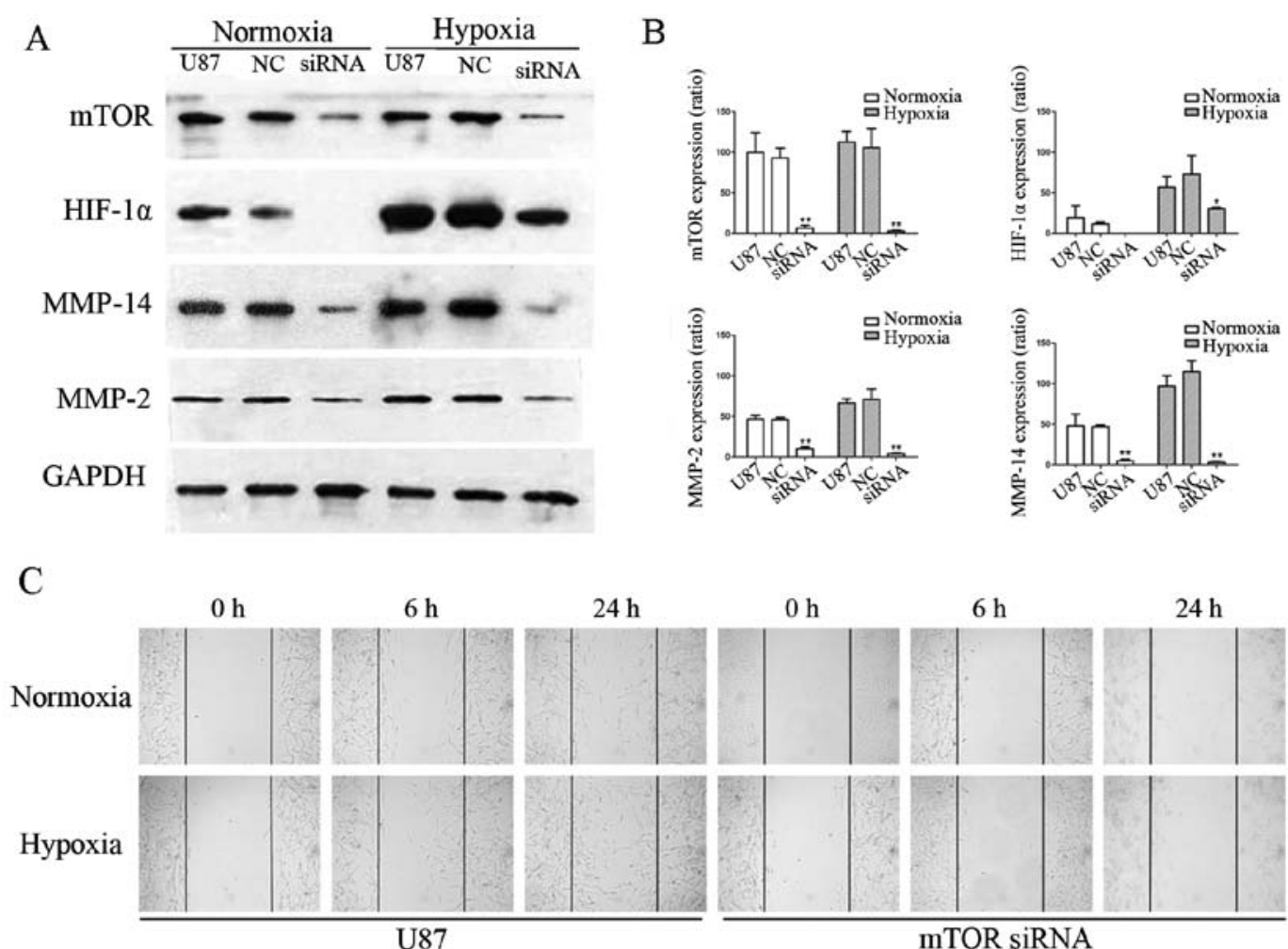

Figure 5. Effect of siRNA on expression of mTOR and downstream molecules, as well as cell migration. (A) Western blotting results for mTOR, HIF-1 $\alpha$, MMP-14 and MMP-2 expression in U87-MG cells with or without interference by siRNA under normoxic and hypoxic conditions for 0-24 h. (B) Quantitative analyses of the western blotting results show the inhibition effects of mTOR siRNA on the expression of related molecules. ${ }^{*} \mathrm{P}<0.05,{ }^{* *} \mathrm{P}<0.01 \mathrm{compared}$ with the U87 group without interference of siRNA. (C) Wound healing assay showed a significant difference between groups with or without interference by mTOR siRNA under normoxic and hypoxic conditions.

However, no relationship was noted with other clinicopathological variants such as gender, age, KPS, or tumor size. Taking all these factors into consideration, VM formed by glioma cells is a novel tumor microcirculation pattern, which is affected by the characteristics inside the tumor cells and outside their microenvironment and differs from classical angiogenesis formed by endothelial cells. Conventional antiangiogenic therapy targeted against tumor vasculature mainly refers to the inhibition of endothelial cells or vascular endothelial growth factor. A previous study found that treatment with bevacizumab (Avastin) is not effective, but also elicits VM formation in tumors to accelerate metastasis, with marked hypoxia (3); thus, targeted therapy for glioma requires other modalities different from the conventional antiangiogenic mechanism, and VM might be a suitable choice.

mTOR, a 289-kDa serine-threonine kinase, is a therapeutic target in glioma (reviewed in ref. 27). Overactivation of the mTOR pathway seems to play an important role in glioma (28). In the present study, the quantity of VM structures increased with the grade of the tumor and the level of mTOR expression. An association between the mTOR signaling pathway and grade of malignancy of human glioma has been noted (29). It is clear that mTOR is a central molecule that controls initiation of protein translation. Decreased oxygen concentration directly affects mTOR signaling and increases the synthesis of HIF-1 $\alpha$ (8). The present study found that rapamycin, a special inhibitor of mTOR, significantly inhibited HIF-1 $\alpha$ accumulation in U87-MG cells, which is similar to the results of other studies from different laboratories, in which hypoxia was induced by a lower oxygen supply $(8,16)$. All these studies provide strong support for the conclusion that mTOR is a positive modulator of the HIF-1 $\alpha$ activation pathway and influences its downstream molecules, including those involved in VM formation.

In recent years, inhibition of signaling molecules involved in VM has become another therapeutic approach for blocking tumor blood supply $(30,31)$. In this study, we found that under normoxic or hypoxic conditions, treatment of U87-MG cells with increasing concentrations of rapamycin induced a dosedependent reduction in tube structures on Matrigel, indicating that rapamycin inhibits VM formation of U87-MG cells even when the VM structures are defective under normoxia. This result gives a more rational option for the clinical treatment of glioma. Some studies have found that specific inhibitors of mTOR have a beneficial effect against gliomas (32-34). However, these studies investigated the roles of rapamycin or its synthetic analogs in tumor proliferation, migration, invasion, and autophagy. In our study, both rapamycin intervention and RNAi confirmed that mTOR is involved in VM formation, which demonstrates a novel role of mTOR in glioma.

Various molecules involved in VM formation have been investigated in different tumors, including HIF-1 $\alpha(14,35)$, VE-cadherin (36,37), EphA2 (37,38), MMP-14 (39), MMP-2 (39) and Ln-5- $\gamma 2$ chain (40). Following the identification of the above-described molecules involved in VM, a classical model of the signaling cascade implicated in VM 
was suggested (reviewed in ref. 10). Pertinent to VM, in this model, hypoxia is initiated to directly modulate EphA2 gene expression (via HIF-1 $\alpha$ ) or to indirectly modulate VE-cadherin expression (via activation of an intermediary protein), consequently promoting the rest of the signaling cascade (41). In our study, consistent with the proposed cascade, inhibition of mTOR was shown to abrogate glioma VM formation. Therefore, we infer that mTOR is involved in VM formation, and is an upstream molecule of HIF-1 $\alpha$. In the final stage of the VM signaling pathway, expression and activation of MMP-14 activates MMP-2. MMP-2 combines with MMP-14 to cleave Ln-5- $\gamma 2$ chain into promigratory fragments. Release of these fragments into the tumor microenvironment can increase migration, invasion, and ultimately result in VM (10). In the present study, we knocked down the mTOR gene and found that both MMP-14 and MMP-2 were decreased under normoxic or hypoxic conditions. The wound healing assay also showed a significant difference between groups with or without interference by siRNA. All of these results confirm that the downstream molecules were influenced by mTOR siRNA. We infer that mTOR signaling is involved in VM formation in gliomas, and inhibition of mTOR can block expression of the downstream molecules in the VM formation signal cascade.

A recent systematic review and meta-analysis showed that VM-positive cancer patients had a poor 5-year overall survival compared with VM-negative malignant tumor cases (42). Thus, treatment targeted against VM seems essential. Given the importance of mTOR as outlined above, if we can find an effective drug against mTOR similar to rapamycin, it may be possible to provide a more rational and effective vascular-targeted therapy for gliomas. In the past 10 years, several agents have been designed to target the mTOR pathway and many other mTOR signaling pathway inhibitors are being studied in clinical trials. Temsirolimus, everolimus, and ridaforolimus are rapalogs that share the same mechanism of action but differ in pharmacokinetic properties because of different substitutions at position C-40 of rapamycin $(27,43)$. The present study found and explained, for the first time, the mechanisms of mTOR participation in the alternative form of tumor blood supply, VM, which provides a novel potential therapeutic target for gliomas. It is noteworthy that mTOR is located at the top of the VM signaling cascade, which can directly sense changes in several signals (such as energy or oxygen level). Therefore, VM treatment targeted against mTOR may be more effective than other downstream molecules. However, the methods we used in our study are mainly in vitro experiments, and animal experiments are needed to confirm the cell-based data. On the other hand, mTOR is a macromolecule $(289 \mathrm{kDa})$ and can be divided into different components (44). Which part is involved specifically in VM formation warrants further study, and any further research will aid our understanding of VM formation.

In conclusion, the present study demonstrated that VM structures are found in highly aggressive glioma tissues by PAS/CD34 double-staining and rapamycin can inhibit VM formation in U87-MG cells under normoxic or hypoxic conditions. We also found that rapamycin and mTOR siRNA can inhibit molecules involved in VM formation via HIF-1 $\alpha$. All of these results indicate that the mTOR signaling pathway is involved in the formation of VM. This study may provide preliminary evidence for a more integrated signaling cascade for $\mathrm{VM}$ formation, and $\mathrm{mTOR}$ is a potential therapeutic target for gliomas.

\section{Acknowledgements}

This research was supported in part by the National Natural Science Foundation of China (NSFC) (81272806 to Y.K.) and (81302199 to X.S.), the Natural Science Foundation of Guangdong Province, China (S2012010009088 to Y.K.) and the Medical Scientific Research Foundation of Guangdong Province, China (B2013246 to X.S.).

\section{References}

1. Ahmadloo N, Kani AA, Mohammadianpanah M, et al: Treatment outcome and prognostic factors of adult glioblastoma multiforme. J Egypt Natl Canc Inst 25: 21-30, 2013.

2. Reardon DA, Herndon JE II, Peters K, et al: Outcome after bevacizumab clinical trial therapy among recurrent grade III malignant glioma patients. J Neurooncol 107: 213-221, 2012.

3. Xu Y, Li Q, Li XY, Yang QY, Xu WW and Liu GL: Short-term anti-vascular endothelial growth factor treatment elicits vasculogenic mimicry formation of tumors to accelerate metastasis. J Exp Clin Cancer Res 31: 16, 2012.

4. Maniotis AJ, Folberg R, Hess A, et al: Vascular channel formation by human melanoma cells in vivo and in vitro: vasculogenic mimicry. Am J Pathol 155: 739-752, 1999.

5. Wang SY, Ke YQ, Lu GH, et al: Vasculogenic mimicry is a prognostic factor for postoperative survival in patients with glioblastoma. J Neurooncol 112: 339-345, 2013.

6. Wang SY, Yu L, Ling GQ, et al: Vasculogenic mimicry and its clinical significance in medulloblastoma. Cancer Biol Ther 13: 341-348, 2012.

7. Karar J and Maity A: PI3K/AKT/mTOR pathway in angiogenesis. Front Mol Neurosci 4: 51, 2011.

8. Land SC and Tee AR: Hypoxia-inducible factor 1alpha is regulated by the mammalian target of rapamycin (mTOR) via an mTOR signaling motif. J Biol Chem 282: 20534-20543, 2007.

9. Su M, Feng YJ, Yao LQ, et al: Plasticity of ovarian cancer cell SKOV3ip and vasculogenic mimicry in vivo. Int J Gynecol Cancer 18: 476-486, 2008.

10. Paulis YW, Soetekouw PM, Verheul HM, Tjan-Heijnen VC and Griffioen AW: Signalling pathways in vasculogenic mimicry. Biochim Biophys Acta 1806: 18-28, 2010.

11. Zhu P, Ning Y, Yao L, Chen M and Xu C: The proliferation, apoptosis, invasion of endothelial-like epithelial ovarian cancer cells induced by hypoxia. J Exp Clin Cancer Res 29: 124, 2010.

12. Snijder B, Sacher R, Ramo P, et al: Single-cell analysis of population context advances RNAi screening at multiple levels. Mol Syst Biol 8: 579, 2012

13. Liu R, Yang K, Meng C, Zhang Z and Xu Y: Vasculogenic mimicry is a marker of poor prognosis in prostate cancer. Cancer Biol Ther 13: 527-533, 2012.

14. Sun W, Shen ZY, Zhang H, et al: Overexpression of HIF-1 $\alpha$ in primary gallbladder carcinoma and its relation to vasculogenic mimicry and unfavourable prognosis. Oncol Rep 27: 1990-2002, 2012.

15. Comito G, Calvani M, Giannoni E, et al: HIF-1 $\alpha$ stabilization by mitochondrial ROS promotes Met-dependent invasive growth and vasculogenic mimicry in melanoma cells. Free Radic Biol Med 51: 893-904, 2011.

16. Abraham RT: mTOR as a positive regulator of tumor cell responses to hypoxia. Curr Top Microbiol Immunol 279: 299-319, 2004.

17. Wang L, Zhang ZG, Zhang RL, et al: Matrix metalloproteinase 2 (MMP2) and MMP9 secreted by erythropoietin-activated endothelial cells promote neural progenitor cell migration. J Neurosci 26: 5996-6003, 2006.

18. Ahmadi SA, Moinfar M, Gohari Moghaddam K and Bahadori M: Practical application of angiogenesis and vasculogenic mimicry in prostatic adenocarcinoma. Arch Iran Med 13: 498-503, 2010.

19. Sharma N, Seftor RE, Seftor EA, et al: Prostatic tumor cell plasticity involves cooperative interactions of distinct phenotypic subpopulations: role in vasculogenic mimicry. Prostate 50: 189-201, 2002. 
20. van der Schaft DW, Hillen F, Pauwels P, et al: Tumor cell plasticity in Ewing sarcoma, an alternative circulatory system stimulated by hypoxia. Cancer Res 65: 11520-11528, 2005.

21. Liu WB, Xu GL, Jia WD, et al: Prognostic significance and mechanisms of patterned matrix vasculogenic mimicry in hepatocellular carcinoma. Med Oncol 28: S228-S238, 2011.

22. Sun T, Sun BC, Zhao XL, et al: Promotion of tumor cell metastasis and vasculogenic mimicry by way of transcription coactivation by Bcl-2 and Twist1: a study of hepatocellular carcinoma. Hepatology 54: 1690-1706, 2011.

23. Baeten CI, Hillen F, Pauwels P, de Bruine AP and Baeten CG: Prognostic role of vasculogenic mimicry in colorectal cancer. Dis Colon Rectum 52: 2028-2035, 2009.

24. Yue WY and Chen ZP: Does vasculogenic mimicry exist in astrocytoma? J Histochem Cytochem 53: 997-1002, 2005.

25. Sun B, Zhang D, Zhang S, Zhang W, Guo H and Zhao X: Hypoxia influences vasculogenic mimicry channel formation and tumor invasion-related protein expression in melanoma. Cancer Lett 249: 188-197, 2007.

26. Zhao N, Sun BC, Sun T, et al: Hypoxia-induced vasculogenic mimicry formation via VE-cadherin regulation by Bcl-2. Med Oncol 29: 3599-3607, 2012.

27. Gomez-Pinillos A and Ferrari AC: mTOR signaling pathway and mTOR inhibitors in cancer therapy. Hematol Oncol Clin North Am 26: 483-505, 2012.

28. Guertin DA and Sabatini DM: Defining the role of mTOR in cancer. Cancer Cell 12: 9-22, 2007.

29. Li XY, Zhang LQ, Zhang XG, et al: Association between $\mathrm{AKT} / \mathrm{mTOR}$ signalling pathway and malignancy grade of human gliomas. J Neurooncol 103: 453-458, 2011.

30. Itzhaki O, Greenberg E, Shalmon B, et al: Nicotinamide inhibits vasculogenic mimicry, an alternative vascularization pathway observed in highly aggressive melanoma. PLoS One 8: e57160, 2013.

31. Serwe A, Rudolph $\mathrm{K}$, Anke T and Erkel G: Inhibition of TGF- $\beta$ signaling, vasculogenic mimicry and proinflammatory gene expression by isoxanthohumol. Invest New Drugs 30: 898-915, 2012.

32. Iwamaru A, Kondo Y, Iwado E, et al: Silencing mammalian target of rapamycin signaling by small interfering RNA enhances rapamycin-induced autophagy in malignant glioma cells. Oncogene 26: 1840-1851, 2007.
33. Li C, Liu Y, Liu J, et al: Rapamycin inhibits human glioma cell proliferation through down-regulating mammalian target of rapamycin pathway and up-regulating microRNA-143. Head Neck Oncol 4: 66, 2012.

34. Heimberger AB, Wang E, McGary EC, et al: Mechanisms of action of rapamycin in gliomas. Neuro Oncol 7: 1-11, 2005.

35. Misra RM, Bajaj MS and Kale VP: Vasculogenic mimicry of HT1080 tumour cells in vivo: critical role of HIF-1 $\alpha$-neuropilin-1 axis. PLoS One 7: e50153, 2012.

36. Hendrix MJ, Seftor EA, Meltzer PS, et al: Expression and functional significance of VE-cadherin in aggressive human melanoma cells: role in vasculogenic mimicry. Proc Natl Acad Sci USA 98: 8018-8023, 2001.

37. Hess AR, Seftor EA, Gruman LM, Kinch MS, Seftor RE and Hendrix MJ: VE-cadherin regulates EphA2 in aggressive melanoma cells through a novel signaling pathway: implications for vasculogenic mimicry. Cancer Biol Ther 5: 228-233, 2006.

38. Chen LX, He YJ, Zhao SZ, et al: Inhibition of tumor growth and vasculogenic mimicry by curcumin through down-regulation of the Eph A2/PI3K/MMP pathway in a murine choroidal melanoma model. Cancer Biol Ther 11: 229-235, 2011.

39. Hess AR, Seftor EA, Seftor RE and Hendrix MJ: Phosphoinositide 3-kinase regulates membrane Type 1-matrix metalloproteinase (MMP) and MMP-2 activity during melanoma cell vasculogenic mimicry. Cancer Res 63: 4757-4762, 2003.

40. Seftor RE, Seftor EA, Kirschmann DA and Hendrix MJ: Targeting the tumor microenvironment with chemically modified tetracyclines: inhibition of laminin 5 gamma2 chain promigratory fragments and vasculogenic mimicry. Mol Cancer Ther 1: $1173-1179,2002$.

41. Kirschmann DA, Seftor EA, Hardy KM, Seftor RE and Hendrix MJ: Molecular pathways: vasculogenic mimicry in tumor cells: diagnostic and therapeutic implications. Clin Cancer Res 18: 2726-2732, 2012.

42. Cao Z, Bao M, Miele L, Sarkar FH, Wang Z and Zhou Q: Tumour vasculogenic mimicry is associated with poor prognosis of human cancer patients: a systemic review and meta-analysis. Eur J Cancer 49: 3914-3923, 2013.

43. Ballou LM and Lin RZ: Rapamycin and mTOR kinase inhibitors. J Chem Biol 1: 27-36, 2008.

44. Laplante M and Sabatini DM: mTOR signaling in growth control and disease. Cell 149: 274-293, 2012. 